\title{
Energetics and electronic structure of encapsulated single-stranded DNA in carbon nanotubes
}

\author{
Katsumasa Kamiya and Susumu Okada \\ Graduate School of Pure and Applied Sciences, University of Tsukuba, 1-1-1 Tennodai, Tsukuba, Ibaraki 305-8571, Japan \\ Japan Science and Technology Agency, CREST, 5 Sanbancho, Chiyoda ku, Tokyo 102-0075, Japan
}

(Received 25 October 2010; published 26 April 2011)

\begin{abstract}
We report total-energy electronic-structure calculations based on density functional theory performed on single-stranded DNA (ssDNA) encapsulated in single-walled carbon nanotubes (SWCNTs). We find that the encapsulation reaction is exothermic for nanotubes with diameters greater than $1.33 \mathrm{~nm}$. The energy gain is calculated to be in the range of $0.8-1.5 \mathrm{eV} / \mathrm{nm}$, depending on tube diameter, base sequences, and ssDNA structure. In optimal ssDNA-SWCNT hybrid-system geometries, the polar groups of ssDNA, i.e. the POH moiety in its backbone, are located adjacent to the wall of the nanotube. The electronic structure of the hybrid system is qualitatively similar to a simple sum of those of an isolated ssDNA molecule and an empty SWCNT. However, detailed analysis of the electronic structure of the hybrid system reveals that the encapsulation of ssDNA into a SWCNT affects the electronic structures of both the ssDNA and the SWCNT.
\end{abstract}

DOI: 10.1103/PhysRevB.83.155444

PACS number(s): 61.48.De, 87.85.jf, 87.14.gk, 71.15.Mb

\section{INTRODUCTION}

In nanometer-scale materials, the atomistic conformation of the material crucially correlates with its electronic properties. Carbon nanotubes (CNTs) are one of the members of this group, whose electronic structures range from semiconductor to metal depending on the atomic arrangement along the circumference of the material. Biomaterials are another group of materials that are well known to have a strong correlation between their function and conformation. A combination of these two representatives is thus expected to exhibit an unusual conformation in each constituent, which would result in a class of the hybrid nanomaterials which has unique electronic and functional variations.

Indeed, the possibility of the encapsulation of singlestranded DNA (ssDNA) in single-walled carbon nanotubes $(\text { SWCNTs })^{1}$ has recently been the subject of research with the goal of broadening potential applications of the hybrid systems to nanotechnologies such as serving as building blocks for electronic devices, electronic DNA sequencing, and gene delivery systems. ${ }^{2-4}$ For instance, DNA transport through a membrane containing nanotubes has been directly observed through fluorescence spectroscopy, ${ }^{5}$ and electrophoretic transport of single-stranded RNA molecules through the nanopores of nanotube-membrane systems has been simulated using molecular dynamics calculations. ${ }^{6}$ Recently, the formation of SWCNT-encapsulated ssDNA has been demonstrated experimentally. ${ }^{7-9}$ Furthermore, the encapsulation of ssDNA in double-walled carbon nanotubes has also been achieved experimentally. ${ }^{10}$

With regard to their potential applications, considerable attention has been devoted to the control of the electronic properties of DNA-CNT hybrid materials by selecting the base sequence of the DNA molecule. For example, SWCNTencapsulated ssDNA could be fabricated to serve as a conducting channel for a field-effect transistor (FET); it has been demonstrated that transport properties of semiconducting SWCNTs can be controlled by the encapsulation of a ssDNA molecule, which has a properly selected base sequence. ${ }^{11}$ In particular, single-stranded polyguanine can change the $p$-type characteristic of pristine SWCNTs into the $n$-type character, while single-stranded polycytosine simply enhances the $p$-type character. These experimental results have created the possibility of the formation of a $p-n$ junction by using the DNA-CNT hybrid systems, which suggests that this system could be a feasible building block for nanoelectronic devices. However, despite the successful fabrication of the ssDNASWCNT hybrid system, its fundamental characteristics are still far from being fully understood.

The purpose of this work is to provide theoretical insight into the energetics, geometry, and electronic structure of the SWCNT-encapsulated ssDNA. Here we simulate isolated chains of ssDNA of infinite length that have been inserted in $(n, 0)$ zigzag nanotubes $(n=16 \ldots .26)$ to probe the properties of these hybrid systems. Total-energy electronic-structure calculations based on density functional theory (DFT) are performed on the models. Our calculations show that the encapsulation reaction of ssDNA is exothermic for CNTs with diameters greater than $1.33 \mathrm{~nm}$, but the energy gain upon encapsulation strongly depends on the tube diameter, base sequences, and ssDNA conformation. The electronic structure of the hybrid systems is qualitatively similar to the sum of those of an isolated ssDNA and an empty SWCNT. However, the encapsulation of ssDNA into a SWCNT quantitatively affects electronic properties of both the ssDNA and the SWCNT. The outcome of this set of calculations shows the unique role of the inner space of the SWCNTs in the control of functionalization of the DNA-CNT hybrid system.

\section{CALCULATION METHODS}

We consider four representative types of ssDNA of infinite length that have repeating base sequences of adenine (poly-A), guanine (poly-G), thymine (poly-T), or cytosine (poly-C). These ssDNA molecules were inserted into $(n, 0)$ zigzag carbon nanotubes $(n=16 \ldots 26)$, which leads to the formation of the ssDNA-SWCNTs hybrid systems. We imposed a commensurability condition between the one-dimensional periodicity of the CNT and repeating periodicity of the ssDNA. Consequently, the lattice parameter $c$ becomes $8.52 \AA$ along the tube direction, corresponding to a double period of the 
zigzag CNT and the double bases of the ssDNA chain. For the lateral direction, we used a square lattice where the system is separated by more than $6 \AA$ from its periodically repeated images to avoid artificial effects. Due to the commensurability condition between CNT and ssDNA, we consider the zigzag carbon nanotube as a host tube for ssDNA. All calculations were performed using DFT. ${ }^{12,13}$ We used the local-density approximation (LDA) to treat the exchange-correlation interactions for electrons. ${ }^{14}$ The LDA is known to well reproduce the layer-layer distance of graphite in agreement with the experimental results, thereby being widely used for carbon related systems. ${ }^{15-18}$ The use of this approximation could thus provide a qualitatively reasonable description of the geometric and electronic structures of ssDNA-SWCNT hybrid-system. Electron-ion interactions were described using TroullierMartins norm-conserving pseudopotentials. ${ }^{19}$ Valence wave functions were expanded in terms of a plane wave basis set with an energy cutoff of $70 \mathrm{Ry}$. Four $k$ points in the one-dimensional Brillouin zone (BZ) were sampled for the BZ integration. During the geometry optimizations, all atoms were relaxed until the residual forces were less than $0.14 \mathrm{eV} / \AA$. The calculations were performed using the CPMD code. ${ }^{20}$

\section{RESULTS AND DISCUSSION}

Figure 1 shows optimized geometries of ssDNA in two different conformations inside the SWCNTs whose diameters are the most appropriate for each base sequence. In these ssDNA conformations, the base planes are approximately parallel or perpendicular to its backbone, which will be referred to as the coating or stacking forms, respectively. In all of the bases, the POH moiety in the backbone of the ssDNA molecule is located in the vicinity of the wall of the nanotube and its hydroxyl group points toward the wall. The calculated distances between the $\mathrm{H}$ atom of the hydroxyl group and the CNT wall are in the range of 1.74-2.36 $\AA$. These distances are close to typical hydrogen bonding lengths; the hydrogen and hydrogen-bonding-acceptor distance in normal hydrogen bonds is in the range of 1.5-2.2 $\AA^{21}$ The smallest distance, $d=1.74 \AA$, is obtained in the case of the coating form of poly-C encapsulated in a $(18,0)$ nanotube (Fig. 1$)$. In this case, the wall of the CNT near the POH moiety is slightly distorted from a perfectly cylindrical shape. However, no significant structural distortion is present in the remaining parts of the system. A similar tendency is observed in the cases for the stacking forms of poly-G, poly-T, and poly-C.

We next investigate encapsulation energy $\Delta E$, which is defined by the following reaction:

$$
(n, 0) \text { tube }+ \text { ssDNA } \rightarrow \text { ssDNA@ }(n, 0)+\Delta E .
$$

In this reaction, total energies for each constituent are calculated on the fully relaxed geometries in vacuum condition. Figure 2 shows the encapsulation energy as a function of the diameter of the SWCNTs. The encapsulation reaction is exothermic for most of the nanotubes. The narrowest CNT diameter for allowing encapsulation is $1.33 \mathrm{~nm}$ for poly-A, poly-G, and poly-C, and $1.41 \mathrm{~nm}$ for poly-T. It was found that the optimal CNT diameter for encapsulation strongly depends on the base sequences. The calculated diameters are $1.48 \mathrm{~nm}$ for poly-A, $1.48 \mathrm{~nm}$ for poly-G, $1.57 \mathrm{~nm}$ for poly-T, (a)
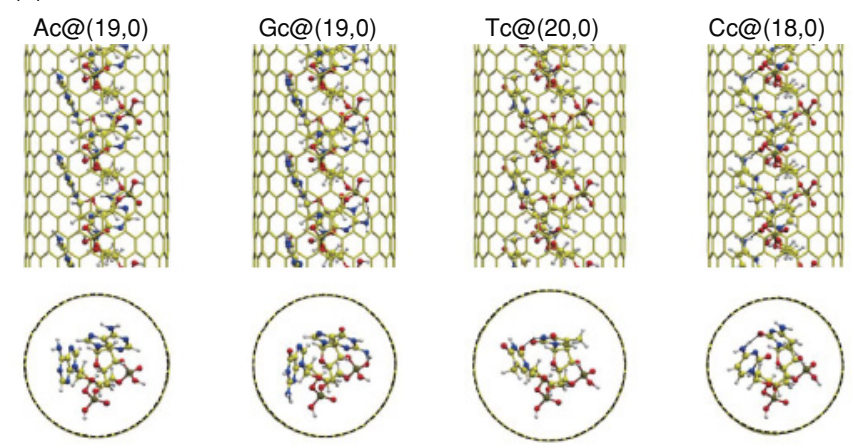

(b)
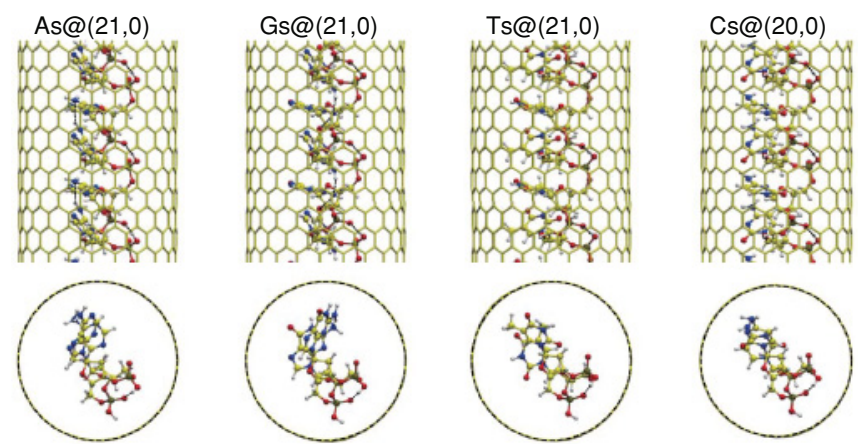

FIG. 1. (Color) Optimized geometries of ssDNA encapsulated in their corresponding energetically optimized nanotubes for the (a) coating and (b) stacking ssDNA configurations. A complete circle is shown by dashed lines, superimposed on nanotubes in the top view panels. Pairs of atoms satisfying the H-bond criteria, i.e., separated by $3.2 \AA$ with a bonding angle of $120^{\circ}$, are connected by dotted lines. Here and in the following figures, the color code for the atoms is yellow for $\mathrm{C}$, white for $\mathrm{H}$, red for $\mathrm{O}$, blue for $\mathrm{N}$, and $\tan$ for $\mathrm{P}$, respectively.

and $1.41 \mathrm{~nm}$ for poly-C in the coating forms, whereas in the case of the stacking form, they are $1.65 \mathrm{~nm}$ for poly-A, $1.65 \mathrm{~nm}$ for poly-G, $1.65 \mathrm{~nm}$ for poly-T, and $1.57 \mathrm{~nm}$ for poly-C. In these optimal CNT configurations, the energy

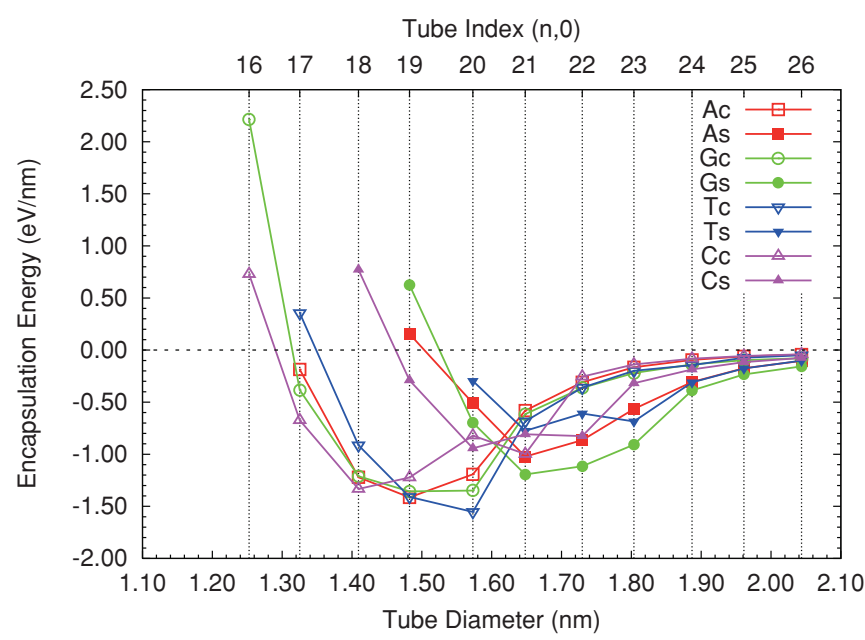

FIG. 2. (Color) Encapsulation energy as a function of tube diameter. 
gain upon encapsulation for each ssDNA molecule is -1.41 , $-1.36,-1.55$, and $-1.33 \mathrm{eV} / \mathrm{nm}$ for the coating forms of poly-A, poly-G, poly-T, and poly-C, respectively, while it is $-1.02,-1.19,-0.78$, and $-0.94 \mathrm{eV} / \mathrm{nm}$ in the case of the stacking forms, respectively. These results provide a clear indication that the energetics depend not only on the space provided by the CNT, but also on the base sequences and conformations of the ssDNA. Based on this base-sequence dependency, SWCNTs could be applied as nanometer-scale molecular filters that would be able to extract isolated bases or homogenous polymers.

In the case of encapsulated-ssDNA conformation, there are two characteristic arrangements inside the SWCNTs, the coating and stacking configurations, which are shown in Fig. 1. In the coating configuration, the base planes of the ssDNA are tilted or parallel with respect to its backbone, while in the stacking configuration, they are aligned perpendicular to the backbone. Because of the difference in size between these two forms, it is expected that the nanometer-scale space inside the CNT could be used to control the conformation of the ssDNA. This would allow the tuning of their functions and electronic structures. Indeed, from Fig. 2, it can be seen that ssDNA in the coating configurations can be inserted into much thinner tubes than the stacking ones, and also have larger encapsulation energies. On the other hand, the stacking arrangement is more favorable for CNTs of larger diameters. This indicates that the stability of these two conformations can be tuned according to the size of the inner space of the SWCNTs. To qualitatively investigate the stability of the ssDNA conformations, we compare the relative stability of these two typical conformations against the tube index $n$ by evaluating the energy difference between the ssDNA-SWCNT systems that have coating and stacking ssDNA, i.e., $\Delta E(n)=$ $E_{\text {coat }}(n)-E_{\text {stack }}(n)$ (Fig. 3). For all of the base sequences, the coating conformation is energetically favorable for the $n \leqslant 20$ CNTs, which have diameters smaller than $1.57 \mathrm{~nm}$. However, the stacking conformation is favorable for the $(22,0)$

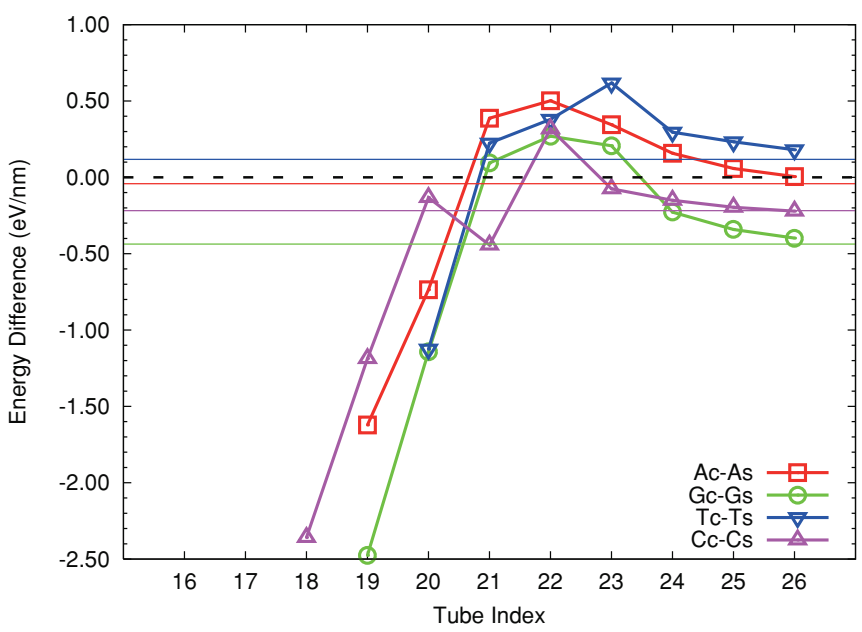

FIG. 3. (Color) Total energy difference between the coating and stacking ssDNA-SWCNT systems, i.e., $\Delta E(n)=E_{\text {coat }}(n)-$ $E_{\text {stack }}(n)$, plotted as a function of the tube index $n$. The red, green, blue, and purple horizontal lines indicate the values obtained for isolated poly-A, poly-G, poly-T, and poly- $\mathrm{C}$ in vacuum, respectively. (a)
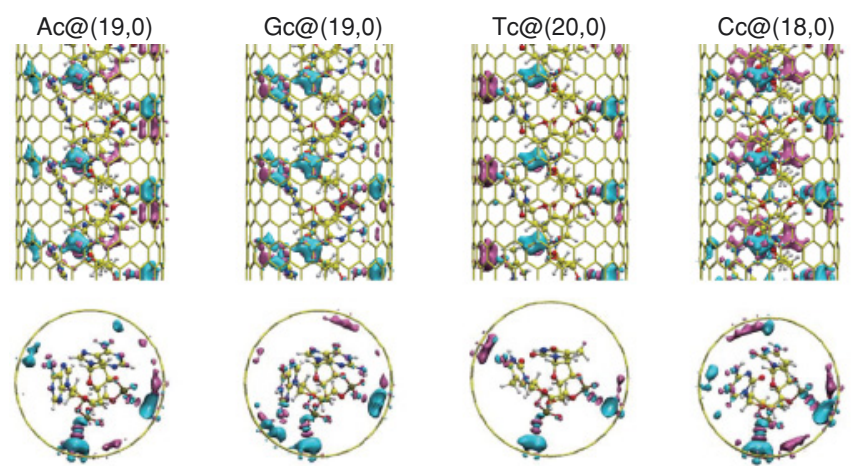

(b)
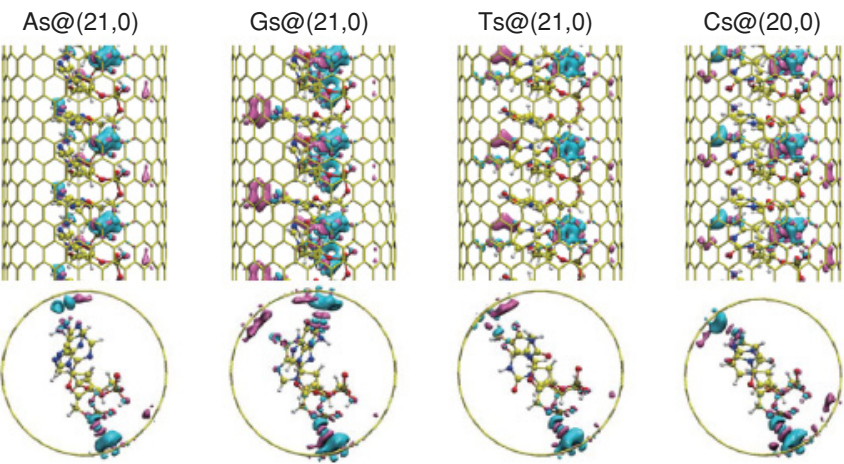

FIG. 4. (Color) Isosurface of the difference between the electron density of the ssDNA-SWCNT hybrid system and the sum of those of each component, i.e., $\Delta \rho(\mathbf{r})=\rho^{\mathrm{sSDNA} @ S W C N T}(\mathbf{r})-\rho^{\mathrm{SWCNT}}(\mathbf{r})-$ $\rho^{\text {ssDNA }}(\mathbf{r})$, for the (a) coating and (b) stacking ssDNA configurations. Blue and pink represent positive and negative values, respectively. The isovalues are $\pm 7.5 \times 10^{-4}\left(e /\right.$ a.u. $\left.^{3}\right)$. The atomic configurations are the same as those of Fig. 1.

CNT, which has a diameter of $1.73 \mathrm{~nm}$ for all of the base sequences. When the diameter is increased further, the stability of these two configurations asymptotically approaches that of the isolated case. These results indicate that the inner space of the SWCNTs controls the stability of this hybrid system and the conformation of the encapsulated ssDNA.

Figure 4 shows the redistribution of the valence charge upon the encapsulation of the ssDNA in the SWCNT that has the optimal diameter for the base sequence of the implanted ssDNA. Electrons collect in the spacious region between the $\mathrm{POH}$ moiety of the ssDNA and the wall of nanotube. This is predominantly caused by the existence of polarized $\mathrm{O}-\mathrm{H}$ bonds near the nanotube wall, where effective attractive potentials are generated by the positively charged $\mathrm{H}$ atoms. The situation is similar in the case of the other polar groups in ssDNA. Specifically, the electrons tend to collect near the $\mathrm{NH}_{2}$ group of the ssDNA bases, while they are repelled from the negatively charged $\mathrm{N}$ and $\mathrm{O}$ atoms (Fig. 4). These results suggest that the interactions between the polar groups, particularly those of the POH moiety in the ssDNA backbone, and the nanotube wall are of great importance. In other words, the interactions between the permanent dipole moment of ssDNA and the induced dipole moment of the CNT wall essentially affect the ssDNA-SWCNT system. 
(a)
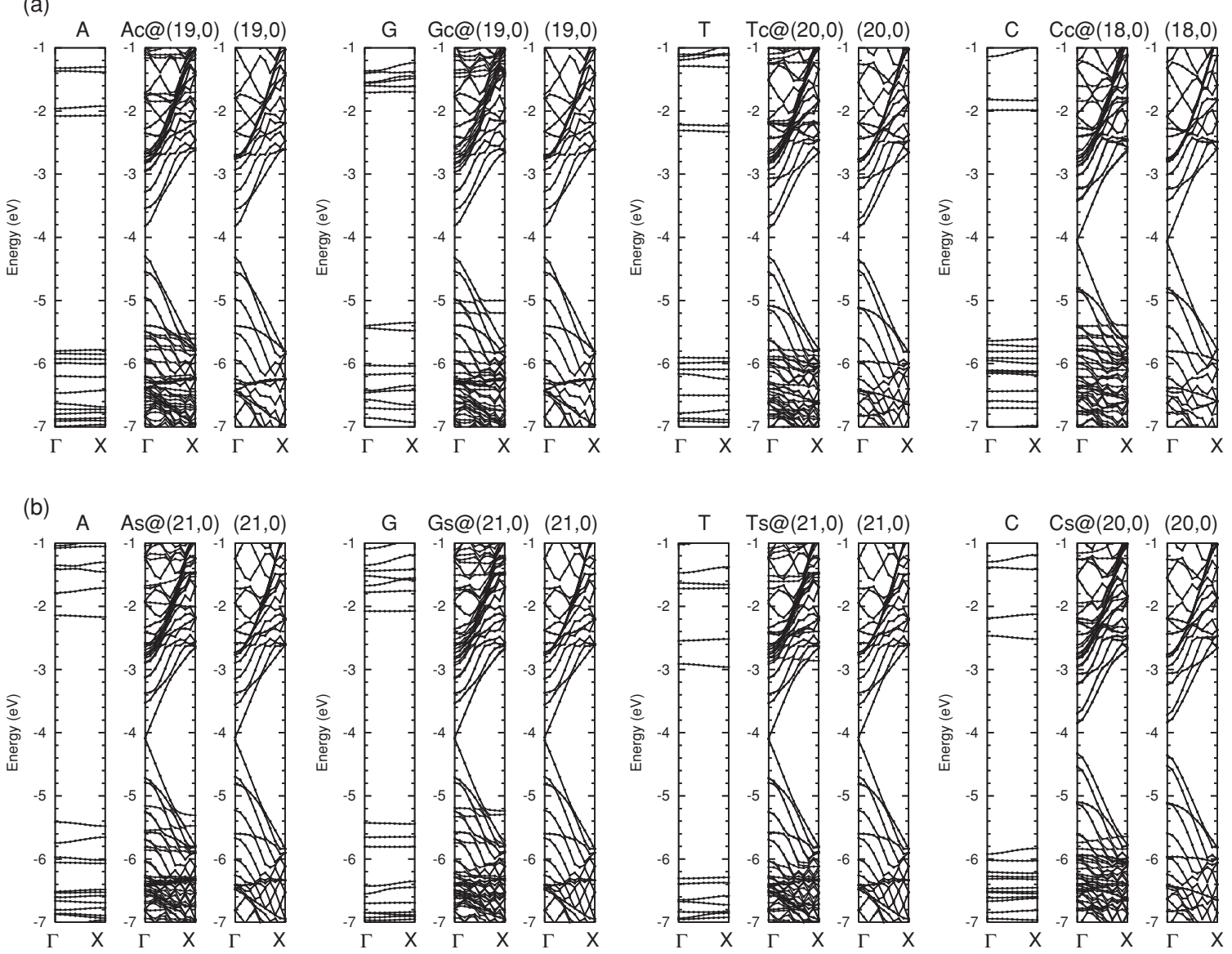

FIG. 5. Energy band structures of the ssDNA-SWCNT hybrid systems, isolated ssDNA, and isolated SWCNTs, for the (a) coating and (b) stacking conformations. Energies are measured from the vacuum levels, which were evaluated from the values of the self-consistent local potential at the edge of the unit cell. The atomic configurations are the same as those of Fig. 1.

We note that a recent study on SWCNT-encapsulated ssDNA based on classical molecular dynamics has shown the importance of both of van der Waals and hydrophobic interactions between the ssDNA bases and the SWCNT. ${ }^{1}$ In addition to these two interactions, our first-principles calculations highlight that the electrostatic interaction induced by the ssDNA backbone is another important factor in determining the energetics of this hybrid system. The existence of such an interaction is supported through a comparison with the SWCNT-encapsulation reaction of $\mathrm{C}_{60}$. As mentioned above, the largest possible energy gain from the SWCNTencapsulation of ssDNA is on the order of $1 \mathrm{eV}$ per nm. This value is comparable with the energy gain upon the SWCNT-encapsulation of $\mathrm{C}_{60} \cdot{ }^{15-18}$ However, the contact area between the ssDNA and the SWCNT is expected to be smaller than that of $\mathrm{C}_{60}$ and the SWCNT, which suggests the existence of another factor in addition to the van der Waals interactions between ssDNA and SWCNT.

To fully understand the behavior of this system it is important to investigate its electronic structure. Figure 5 shows the electronic energy bands of ssDNA in the coating and stacking configurations encapsulated in a SWCNT of optimal diameter. The energy band structures of isolated ssDNA and empty SWCNTs are also shown in the same figure. All of the hybrid systems are semiconductors with a direct gap originating from the $E_{11}$ gap of the semiconducting SWCNTs and from the tiny gap associated with the curvature effect in the metallic SWCNTs. ${ }^{22}$ It was found that the energy bands near the Fermi level originate primarily from those of the empty nanotubes. The energy bands associated with ssDNA levels do not appear near the energy gap but emerge as occupied and unoccupied states below $-5 \mathrm{eV}$ and above $-3 \mathrm{eV}$ relative to the vacuum level, respectively. Interestingly, these bands shift upward upon encapsulation regardless of the ssDNA conformation and base sequence. This is predominantly caused by a change in average potential upon the encapsulation of the ssDNA chains in the nanometer spacing of the SWCNTs.

A detailed analysis of the electronic structure can clarify the interesting alterations to the electronic structures of both ssDNA and SWCNT. We first consider the ionization potential of the ssDNA. In general, the guanine base has the shallowest ionization potential among the four bases species, which indicates that the electronic properties of DNA depend on this base sequence. ${ }^{24}$ In addition to its base sequence dependence, we find that the ionization potential for the bases also depends on the size of the inner space of the nanotubes. Figure 6 shows the eigenvalues of the highest occupied and the lowest unoccupied states of the ssDNA molecule at the $\Gamma$ point as a function of the tube index. It can be seen that the guanine base has the shallowest ionization potential among the four bases 


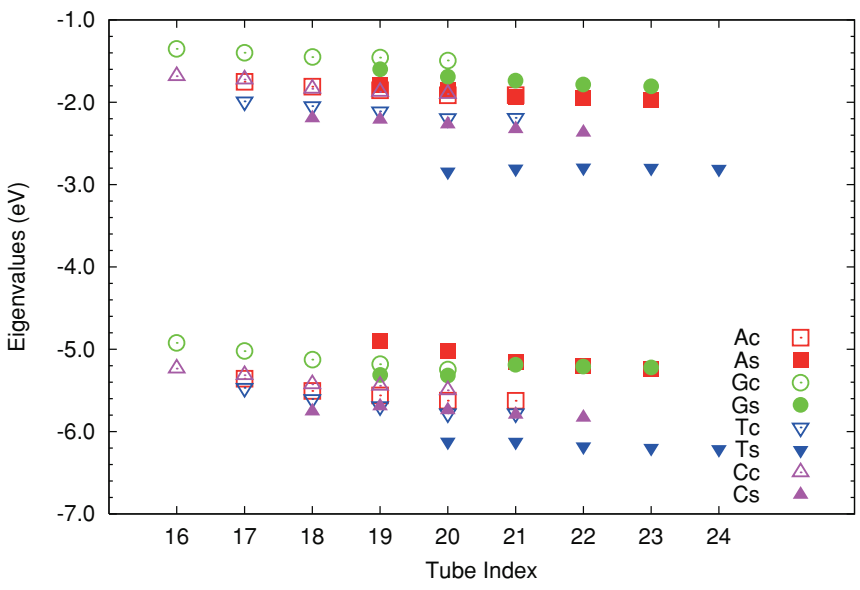

FIG. 6. (Color) Eigenvalues at the $\Gamma$ point of the highest occupied and lowest unoccupied Kohn-Sham states, which originate from the ssDNA molecule, as a function of the tube index $n$. Energies are measured from the vacuum levels.

inside thin nanotubes, while in nanotubes of large diameter, the adenine base has a similar ionization potential to the guanine base. This feature arises from conformation effects in the ssDNA upon the SWCNT encapsulation. Indeed, our calculations performed on isolated ssDNA molecules with coating and stacking conformations show that the order of the ionization potentials is $\mathrm{G}(5.40 \mathrm{eV})<\mathrm{C}(5.64 \mathrm{eV})<$ A $(5.80 \mathrm{eV})<\mathrm{T}(5.90 \mathrm{eV})$ for the coating configuration, whereas it is $\mathrm{A}(5.41 \mathrm{eV})<\mathrm{G}(5.43 \mathrm{eV})<\mathrm{C}(5.92 \mathrm{eV})<$ $\mathrm{T}(6.31 \mathrm{eV})$ for the stacking configuration. These results suggest that electronic properties of ssDNA are altered when it is encapsulated in a SWCNT. Therefore, the nanometerscale space of the SWCNT can control the conformation of the ssDNA, which allows the tuning of its electronic properties.

Let us next consider the electronic properties of the host SWCNT. Figure 7 shows the energy gap modulation in the SWCNT when it encapsulates ssDNA molecules of various base sequences and conformations. This modulation is defined

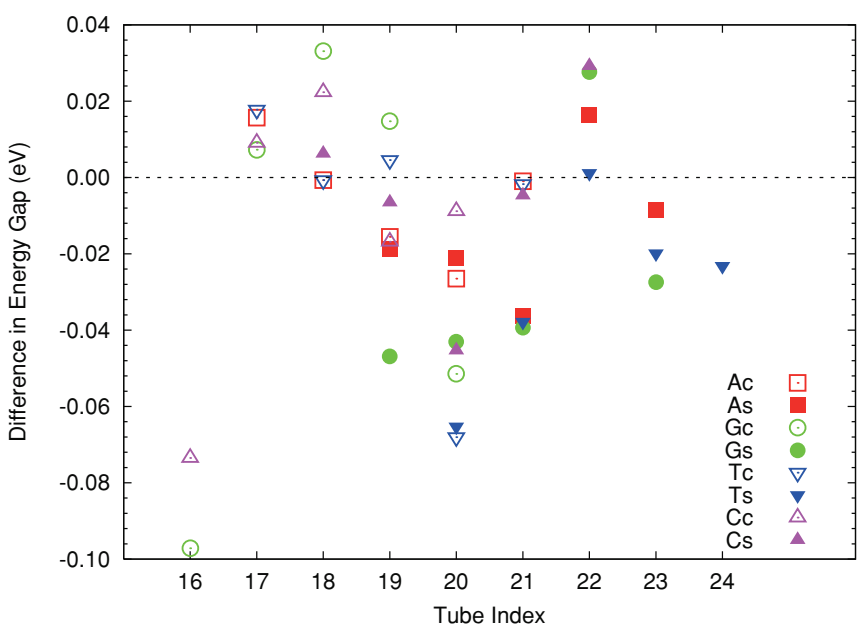

FIG. 7. (Color) Difference in the energy gap between the ssDNA-SWCNT hybrid systems and isolated SWCNTs, i.e., $\Delta E_{\text {gap }}=$ $E_{\text {gap }}^{\text {sSDNA@SWCNT }}-E_{\text {gap }}^{\text {SWCNT }}$, plotted versus the tube index $n$.

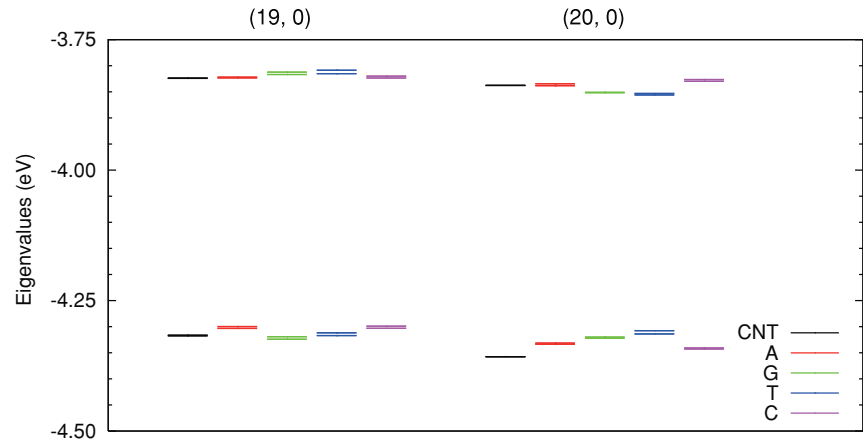

FIG. 8. (Color) Eigenvalues at the $\Gamma$ point of the highest occupied and lowest unoccupied Kohn-Sham states for the $n=19$ and 20 semiconducting nanotubes with encapsulated ssDNA. Energies are measured from the vacuum levels.

as $\Delta E_{\text {gap }}=E_{\text {gap }}^{\text {sSDA } @ S W C N T}-E_{\text {gap }}^{\mathrm{SWCNT}}$. It was found that the energy gap of a SWCNT encapsulating a ssDNA molecule is modulated by a few tens of meV. However, the exact value depends on the base sequences and conformations of the ssDNA molecule. In sharp contrast to the $\mathrm{C}_{60}$ encapsulated in CNTs, ${ }^{23}$ the gap modulation does not exhibit a distinct family pattern dependence. However, for the SWCNT, ssDNA encapsulation leads to gap modulation that is base sequence dependent. This indicates that the identification of base sequences may be possible by monitoring the energy gap of the SWCNT instead of direct observation on the DNA properties.

In addition to the modulation of the energy gap of SWCNTs, there is a change in the absolute eigenvalues that describe the SWCNT $E_{11}$ gap. Figure 8 shows the top of the valence band and the bottom of the conduction band for $(19,0)$ and $(20,0)$ SWCNTs. In the case of the $(20,0)$ CNT, the valence band top for the DNA-SWCNT system is lowered by a few tens of meV compared with that of the empty CNT. Furthermore, among the four kinds of ssDNA, SWCNTencapsulated poly-T possesses the shallowest level, while poly-C has the deepest one. On the other hand, the valence top of the $(19,0)$-SWCNT-encapsulated poly-G was found to be the deepest level, whereas that of poly-C becomes the shallowest. Thus, the occupied states of SWCNT-encapsulated DNA exhibit significant modulations that depend on the base sequence. For the unoccupied states, a base dependency similar to that of the occupied states is found in each case. Note that the base dependence of the absolute eigenvalues of the DNA-SWCNT system could be one of the key factors in the conduction properties of a FET made from this hybrid; these FETs have been experimentally shown to have a basesequence dependency. ${ }^{11}$

\section{CONCLUSION}

We have investigated the energetics and the electronic structures of SWCNT-encapsulated ssDNA by using firstprinciples total-energy calculations based on DFT. These calculations have indicated that the encapsulation reaction is exothermic for nanotubes of diameters greater than $1.33 \mathrm{~nm}$. The energy gain upon DNA encapsulation is in the range of $0.8-1.5 \mathrm{eV} / \mathrm{nm}$ and depends on tube diameter, base sequences, and ssDNA structures. In energetically stable geometries of 
the ssDNA-SWCNT hybrid systems, the polar groups of the ssDNA, i.e., the POH moiety in its backbone, are located in the vicinity of the nanotube wall. Although the electronic structure of the hybrid system is similar to a simple sum of the electronic energy bands of an isolated ssDNA molecule and an empty SWCNT, the encapsulation leads to the significant changes in the electronic structure of each constituent unit. The $E_{11}$ energy gap of the SWCNT is shifted by a few tens of meV relative to that of the empty SWCNT, and the depth of the eigenvalues of the highest occupied and lowest unoccupied states of DNA is also shifted. This study highlights the functional importance of the nanoscale inner space of the SWCNTs for controlling behavior of the encapsulated ssDNA, which raises the possibility of DNA-nanotube-based technologies.

\section{ACKNOWLEDGMENTS}

Computations were performed on a NEC SX-9 at the Institute for Solid-State Physics, University of Tokyo; a Fujitsu PrimeQuest at the Research Center for Computational Science, Okazaki Research Facilities, National Institutes of Natural Sciences; a NEC SX-8/4B at the University of Tsukuba; a NEC SX-8 at the Yukawa Institute of Theoretical Physics, Kyoto University; and a NEC SX-9 at the Information Synergy Center, Tohoku University. This research was supported by a Grant-in-Aid for Young Scientists (B) (No. 22740259), in part by CREST, the Japan Science and Technology Agency, and a Grant-in-Aid for Scientific Research from the Ministry of Education, Culture, Sports, Science, and Technology of Japan.
${ }^{1}$ H. Gao, Y. Kong, D. Cui, and C. S. Ozkan, Nano Lett. 3, 471 (2003).

${ }^{2}$ D. Tasis, N. Tagmatarchis, A. Bianco, and M. Prato, Chem. Rev. 106, 1105 (2006).

${ }^{3}$ A. Bianco, R. Sainz, S. Li, H. Dumortier, L. Lacerda, K. Kostarelos, S. Giordani, and M. Prato, Medicinal Chemistry and Pharmacological Potential of Fullerenes and Carbon Nanotubes (Springer, Dordrecht, 2008).

${ }^{4}$ S. Meng and E. Kaxiras, Biosensing Using Nanomaterials (John Wiley and Sons, Inc., Hoboken, 2009).

${ }^{5}$ T. Ito, L. Sun, and R. M. Crooks, Chem. Commun. 7, 1482 (2003).

${ }^{6}$ I. Yeh and G. Hummer, Proc. Natl. Acad. Sci. USA 101, 12177 (2004).

${ }^{7}$ T. Okada, T. Kaneko, R. Hatakeyama, and K. Tohji, Chem. Phys. Lett. 417, 288 (2006).

${ }^{8}$ T. Kaneko, T. Okada, and R. Hatakeyama, Contrib. Plasma Phys. 47, 57 (2007).

${ }^{9}$ Y. Maruyama, S. Bandow, and S. Motohashi, in Advances in Nanotechnology, edited by Z. Bartul and J. Trenor, Vol. 4 (Nova Science Publishers, Inc., New York, 2010).

${ }^{10}$ Y. Li, T. Kaneko, and R. Hatakeyama, Small 6, 729 (2010).

${ }^{11}$ T. Kaneko and R. Hatakeyama, Applied Physics Express 2, 127001 (2009).
${ }^{12}$ P. Hohenberg and W. Kohn, Phys. Rev. 136, B864 (1964).

${ }^{13}$ W. Kohn and L. J. Sham, Phys. Rev. 140, A1133 (1965).

${ }^{14}$ S. Goedecker, M. Teter, and J. Hutter, Phys. Rev. B 54, 1703 (1996).

${ }^{15}$ S. Okada, Phys. Rev. B 77, 235419 (2008).

${ }^{16}$ S. Okada, S. Saito, and A. Oshiyama, Phys. Rev. Lett. 86, 3835 (2001).

${ }^{17}$ S. Okada, M. Otani, and A. Oshiyama, Phys. Rev. B 67, 205411 (2003).

${ }^{18}$ M. Otani, S. Okada, and A. Oshiyama, Phys. Rev. B 68, 125424 (2003).

${ }^{19}$ N. Troullier and J. L. Martins, Phys. Rev. B 43, 1993 (1991).

${ }^{20}$ CPMD, copyright IBM Corp. 1990-2008, copyright MPI für Festkörperforschung Stuttgart 1997-2001.

${ }^{21} \mathrm{G}$. A. Jeffrey, An Introduction to Hydrogen Bonding (Oxford University Press, Oxford, 1997).

${ }^{22}$ N. Hamada, S. I. Sawada, and A. Oshiyama, Phys. Rev. Lett. 68, 1579 (1992).

${ }^{23}$ T. Okazaki, S. Okubo, T. Nakanishi, T. Saito, M. Otani, S. Okada, S. Bandow, and S. Iijima, J. Am. Chem. Soc. 130, 4122 (2008).

${ }^{24}$ X. Yang, X. Wang, E. R. Vorpagel, and L. Wang, Proc. Natl. Acad. Sci. USA 101, 17588 (2004). 